\title{
Nanoparticles-Free Fluorescence Anisotropy Amplification Assay for Detection of RNA Nucleotide-Cleaving DNAzyme Activity
}

\author{
Dapeng Zhang, ${ }^{\dagger}$ Rong Fu, ${ }^{\dagger}$ Qiang Zhao, ${ }^{\dagger}$ Haiqin Rong, ${ }^{\dagger}$ and Hailin Wang* ${ }^{*}$ \\ ${ }^{\dagger}$ State Key Laboratory of Environmental Chemistry and Ecotoxicology, Research Center for Eco-Environmental Sciences, Chinese \\ Academy of Sciences, Beijing 100085, People's Republic of China \\ ${ }^{\ddagger}$ School of Medicine and Life Sciences, University of Jinan-Shangdong Academy of Medical Sciences, Jinan 250062, People’s Republic \\ of China
}

ABSTRACT: Fluorescence anisotropy is a homogeneous, sensitive, ratiometric, and real-time analytical technology. However, it is a great challenge to produce a large fluorescence anisotropy change upon the presence of target small molecules without nanoparticles-dependent amplification. This work reports a nanoparticle-free and multiple G-enhanced fluorescence anisotropy assay for detection of DNAzyme activity. $\mathrm{A} \mathrm{Pb}^{2+}$-dependent GR-5 DNAzyme was used as a model. We hybridized the rA-cleavable substrate strand containing a TMR label at the 5 '-end with the DNAzyme strand containing an

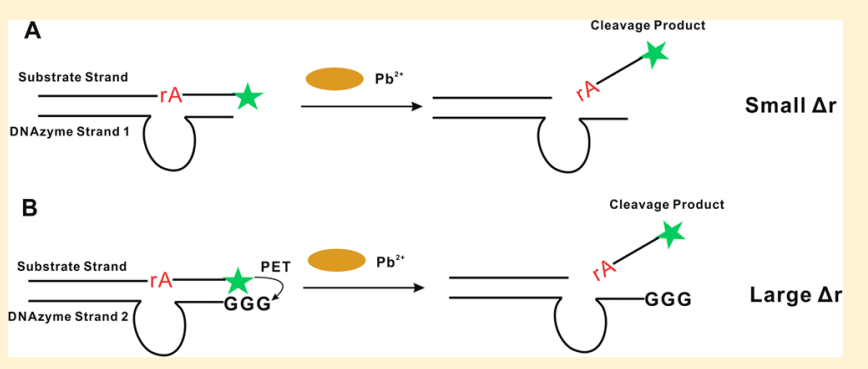
extended three $\mathrm{G}$ bases at the $3^{\prime}$-end. By this design, we demonstrate that both fluorescence quenching and the enhanced DNAzyme activity contribute to a $\mathrm{Pb}^{2+}$-induced large fluorescence anisotropy change $(|\Delta r|=0.168)$. The limit of detection for $\mathrm{Pb}^{2+}$ is estimated to be about $100 \mathrm{pM}$ with a dynamic range from $200 \mathrm{pM}$ to $100 \mathrm{nM}$. The interference from the other nine divalent metal ions of 1000-times excess amount is negligible. Moreover, we show an extended assay for evaluation of the interactions of $\mathrm{Pb}^{2+}$ with cysteine and glutathione by the detection of GR5 DNAzyme activity. Collectively, we developed a novel fluorescence anisotropy amplification assay, enabling us to detect DNAzyme activity and associated cofactors and inhibitors and to characterize the $\mathrm{Pb}^{2+}$-chelation capability of free thiols.

$\mathrm{O}$ ne class of functional nucleic acids with catalytic activity is DNAzymes, which have potential applications in diagnosis, therapeutics, and bioanalysis. ${ }^{1-4}$ Various DNAzymes have been in vitro selected to catalyze a wide range of reactions, such as RNA cleavage, DNA cleavage, RNA ligation, DNA ligation, DNA phosphorylation, cleavage of the phosphoramidate bond, and porphyrin metalation. ${ }^{5,6}$ One type of DNAzymes can cleave RNA. These RNA-cleaving DNAzymes are composed of a DNA enzyme strand and a DNA substrate strand containing a single RNA linkage (rA), which serves as the cleavage site. ${ }^{7}$ This class of DNAzymes has been applied in nucleic acid detection, ${ }^{8}$ metal ions sensing, ${ }^{3,4,6,7,9-11}$ and molecular logic gate constructing. $4,12,13$

The enzymatic activities play central roles in any extended analytical applications of DNAzymes. A number of biochemical and biophysical methods have been carried out for measuring DNAzyme activities, especially for RNA-cleaving DNAzymes. These methods include polyacrylamide gel electrophoresis (PAGE), ${ }^{14}$ MALDI-TOF mass spectrometry, ${ }^{15}$ fluorescence resonance energy transfer (FRET), ${ }^{16,17}$ and fluorescence anisotropy (FA). ${ }^{18-22}$

Among known techniques for detection of DNAzyme activity, FA has a number of advantages. It is a simple, realtime, and truly homogeneous analysis. Moreover, it does not require the separation of substrates and the cleaved products. This technique also presents several other attractive features, such as the requirement of only one fluorescent label and relative insensitivity to the photobleaching of the fluorophore and to instrument parameters due to its ratiometric nature. In fact, FA has been widely applied to immunoassays, single nucleotide polymorphism (SNP) analysis, DNA-protein interaction assays, protein/peptide-ligand binding assays, and enzyme activity assays. ${ }^{23-29}$ In principle, FA is used to measure the rotational motion of a fluorescent molecule-linked object, including global and segmental rotation. However, FA is often used to sense the changes depending on the molecular size/ mass or comparably global rotation according to the Perrin equation. $^{30}$ Interestingly, $\mathrm{FA}$ is also extended to measure nucleic acid conformational changes (e.g., DNA wrapping), ${ }^{29,31}$ and interaction distance ${ }^{32}$ of the conjugated fluorophore. In these cases, the segmental rotation of the labeled fluorescent tag may play more important roles. FA-based DNAzyme assays have been described thus far, ${ }^{18-22}$ but the reported sensing systems usually display a small FA change $(|\Delta r|<0.10)$ due to the small change in molecular size linking to DNAzyme activity. To improve FA change caused by the target small molecules in FA-based DNAzyme assays, nanoparticles (e.g., nanoAu, graphene oxide) are used to enhance the FA response. ${ }^{18,19,21,22}$

By the involvement of nanoparticle-dependent amplification,

Received: February 4, 2015

Accepted: April 11, 2015

Published: April 11, 2015 
Scheme 1. Schematic Representation of the Guanine Quenching-Enhanced FA Assay for Detection of DNAzyme Activity
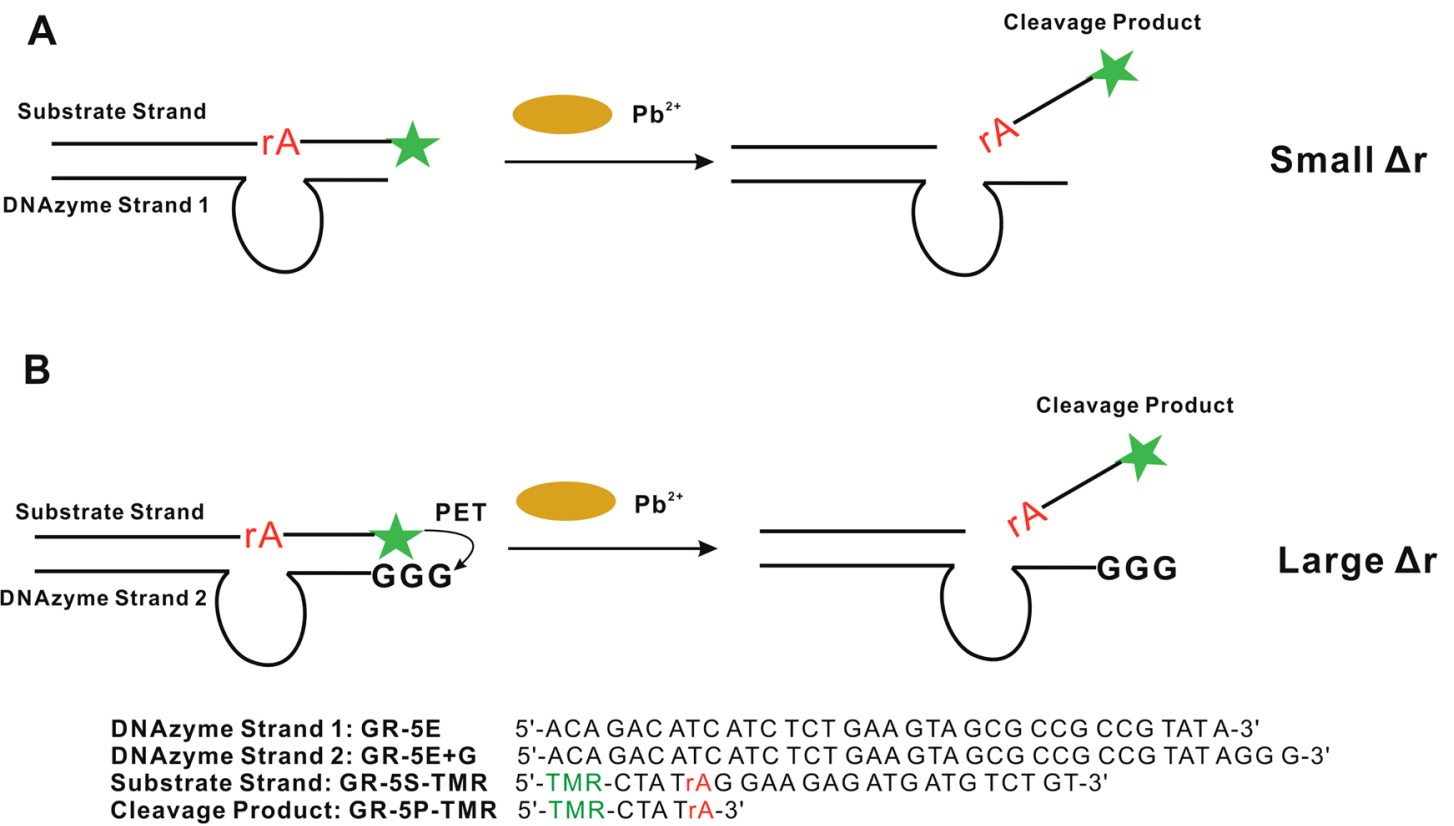

FA changes $(|\Delta r|)$ can increase to $0.08-0.30$ due to a dramatic change in mass/size. So far, it is a great challenge to produce a large fluorescence anisotropy change upon the presence of target small molecules without nanoparticle-like supermoleculedependent amplification.

In this work, by referring to the structural feature of DNAzymes, we designed and examined a labeled tetramethylrhodamine (TMR)-guanine $(\mathrm{G})$ interaction strategy to develop a novel FA-based DNAzyme assay. By taking advantage of binding-induced change of the TMR $-\mathrm{G}$ interaction, recently we demonstrated a series of enhanced FA assays for nucleic acid aptamer affinity analysis. This new FA strategy allows for analysis of proteins and small molecules, showing high sensitivity and selectivity. ${ }^{33-36}$ However, we do not know whether and how this strategy is effective for detection of DNAzyme activity. Here, we took $\mathrm{Pb}^{2+}$-dependent DNAzyme (GR-5) as an example (Scheme 1). In GR-5 DNAzyme, a DNAzyme strand is hybridized with a rA cleavage sitecontaining substrate strand. ${ }^{37,38}$ The $\mathrm{Pb}^{2+}$ binding can stimulate the cleaving activity of the GR-5 DNAzyme. By the cleaving activity, the TMR-labeled substrate is split into two short DNA fragments. To obtain an enhanced FA change, we labeled the TMR at the $5^{\prime}$-end of the substrate strand and attached tandem guanine bases at the $3^{\prime}$-end of enzyme strand. Due to the cleaving activity of the DNAzyme, one shorter fragment cleaved

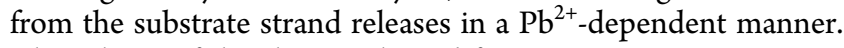
The release of the shorter cleaved fragment containing a TMR label will generate a very low FA response due to the diminishing of the TMR-G interaction. The remarkable FA change could be used to monitor DNAzyme activity. Moreover, by monitoring the DNAzyme activity, the cofactors (e.g, $\mathrm{Pb}^{2+}$ for GR-5 DNAzyme) and their interactions with free thiolcontaining biomolecule can be detected or characterized.

\section{EXPERIMENTAL SECTION}

Materials and Reagent. The sequences of the oligonucleotides used in this study are listed in Table 1 . They were synthesized and purified (HPLC) by Sangon Biotech Co. Ltd. (Shanghai, China) or Takara Biotechnology Co. Ltd. (Dalian,
Table 1. Name and Sequence of Oligonucleotides Used in the Study

\begin{tabular}{ll}
\hline name & Sequence (5' $\left.\rightarrow 3^{\prime}\right)$ \\
\hline GR-5E+3G & 5'-ACA GAC ATC ATC TCT GAA GTA GCG CCG CCG TAT AGG G-3' \\
GR-5E & 5'-ACA GAC ATC ATC TCT GAA GTA GCG CCG CCG TAT A-3' \\
GR-5E+1G & 5'-ACA GAC ATC ATC TCT GAA GTA GCG CCG CCG TAT AG-3' \\
GR-5E+2G & 5'-ACA GAC ATC ATC TCT GAA GTA GCG CCG CCG TAT AGG-3' \\
GR-5E+4G & 5'-ACA GAC ATC ATC TCT GAA GTA GCG CCG CCG TAT AGG GG-3' \\
GR-5E+5G & 5'-ACA GAC ATC ATC TCT GAA GTA GCG CCG CCG TAT AGG GGG-3' \\
GR-5S-TMR & 5'-TMR-CTA TrAG GAA GAG ATG ATG TCT GT-3' \\
GR-5P-TMR & 5'-TMR-CTA TrA-3' \\
\hline
\end{tabular}

${ }^{a}$ Note: The cleavage site of the substrate is shown in boldface type (rA). The red characters represent the fluorescent labeled dye 6carboxytetramethyrhodamine (TMR).

China), some of which were fluorescently labeled with TMR at the 5 -terminal of the substrate strand. The oligonucleotide stock solutions were prepared in ultrapure water and then stocked at $-20{ }^{\circ} \mathrm{C}$. The concentration of the oligonucleotide was represented as a single-stranded concentration, which was determined by measuring the absorbance at $260 \mathrm{~nm}$ using the Thermo Scientific Nanodrop (Wilmington, DE, USA). Pb$\left(\mathrm{NO}_{3}\right)_{2}$ and acetate acid were purchased from National Pharmaceutical Group Chemical Reagent Co. (Beijing, China). Tris(hydroxymethyl)aminomethane (Tris) was purchased from Amresco (Solon, OH, USA). All other reagents were analytical grade and obtained from National Pharmaceutical Group, which were used as received without further purification. Ultrapure water with an electrical resistivity of 18.2 $\mathrm{M} \Omega \cdot \mathrm{cm}$ was obtained from a Purelab Ultra Elga Lab water system (VWS Ltd., High Wycombe, Bucks, U.K.).

FA Measurement. FA analysis was performed on a Fluoromax-4 spectrometer (Horiba Jobin Yvon. Edison, NJ, USA), ${ }^{33-35}$ which measured FA by the L-format configuration. A $150 \mu \mathrm{L}$ quartz cuvette was used for all measurements. The excitation and emission wavelengths were set at 555 and 580 $\mathrm{nm}$, and their corresponding slit bandwidths were set at 5 and $10 \mathrm{~nm}$, respectively. The integration time was $0.5 \mathrm{~s}$. The 
background signal of the buffer solution was subtracted from the collected data for FA calculation. The FA at $580 \mathrm{~nm}$ was used for quantitative analysis.

Detection of $\mathrm{Pb}^{2+}$ and Free Thiol-Containing Biomolecules. DNAzyme assays were performed with $20 \mathrm{nM}$ GR$5 \mathrm{E}+3 \mathrm{G}$ or GR-5E and $20 \mathrm{nM}$ GR-5S-TMR in $50 \mathrm{mM}$ Tris-HAc ( $\mathrm{pH} 7.5)$ containing $200 \mathrm{mM} \mathrm{NaCl}$. The DNA solution was heated to $95{ }^{\circ} \mathrm{C}$ for $5 \mathrm{~min}$ and subsequently cooled to room temperature slowly. Then a different concentration of $\mathrm{Pb}^{2+}$ was added to this hybridized DNAzyme solution. The mixture was allowed to incubate at room temperature for $120 \mathrm{~min}$. FA of the reaction mixture was recorded for quantitative analysis.

For the detection of free thiol-containing biomolecules, different concentration of cysteine or glutathione was first added to this hybridized DNAzyme solution, and the mixture was incubated at room temperature for $30 \mathrm{~min}$. Fixed $100 \mathrm{nM}$ $\mathrm{Pb}^{2+}$ was then added, and the mixture was incubated at room temperature for $120 \mathrm{~min}$ before FA measurement.

\section{RESULTS AND DISCUSSION}

Detection of GR-5 DNAzyme Activity by Fluorescence Anisotropy. As illustrated in Scheme 1, we designed two types of GR-5 DNAzymes. Both were fluorescently labeled at the 5'end of the substrate strand (GR-5S-TMR) with only one fluorophore TMR. In particular, the 3 '-end of the DNAzyme strand was extended with three tandem $G$ bases in the second hybridized DNAzyme (GR-5E+3G/GR-5S-TMR, Scheme 1B). This allows the labeled TMR to interact with the three tandem $\mathrm{G}$ bases in the second hybridized DNAzyme. In contrast, to the first hybridized DNAzyme (GR-5E/GR-5S-TMR, Scheme 1A), there is no strong interaction between the labeled TMR and G bases due to the absence of the tandem $G$ bases at the $3^{\prime}$-end of the DNAzyme strand. In the presence of specific cofactor $\mathrm{Pb}^{2+}$, both DNAzymes can catalyze the irreversible cleavage of the substrate strand at the rA site as two fragments of 18 nucleotides and five nucleotides in length, respectively. The cleavage causes a dissociation of the TMR-labeled fragment of five nucleotides from the hybridized DNAzymes due to a limited number of base pairing ( $5 b p)$. Then, the shortened and dissociated TMR-labeled substrate fragment should show a decreased FA due to its reduced molecular size accelerating diffusional rotation. Thus, this FA decrease strategy is used to monitor the DNAzyme activity.

It is important that, in the second DNAzyme (GR-5E+3G/ GR-5S-TMR), the labeled TMR could interact with tandem G bases in the complementary strand. By this interaction via photoinduced electron transfer (PET) mechanism, ${ }^{39-43}$ the fluorescence quenching and the decreased fluorescence lifetime of the labeled TMR in the GR-5E+3G/GR-5S-TMR are expected. Therefore, the labeled TMR should display a large FA value in this DNAzyme. Once the interaction between TMR and the adjacent guanine base is diminished after the substrate strand is cleaved into two dissociable segments, a considerable decrease in FA of the labeled TMR can be found.

Indeed, we observed a larger FA change for GR-5E+3G/GR5S-TMR $(\Delta r=-0.163)$, which is about 2-folds as high as that for GR-5E/GR-5S-TMR $(\Delta r=-0.088)$ upon the presence of excess $\mathrm{Pb}^{2+}$ (Figure 1). In detail, the FA value $(r)$ of the substrate GR-5S-TMR was found to be approximately 0.137 . When this substrate strand GR-5S-TMR was hybridized with the equal amount of the DNAzyme strand GR-5E, the FA value $(r)$ increased to 0.210 (Figure 1). While it was hybridized with the DNAzyme strand containing three extended G bases (GR-

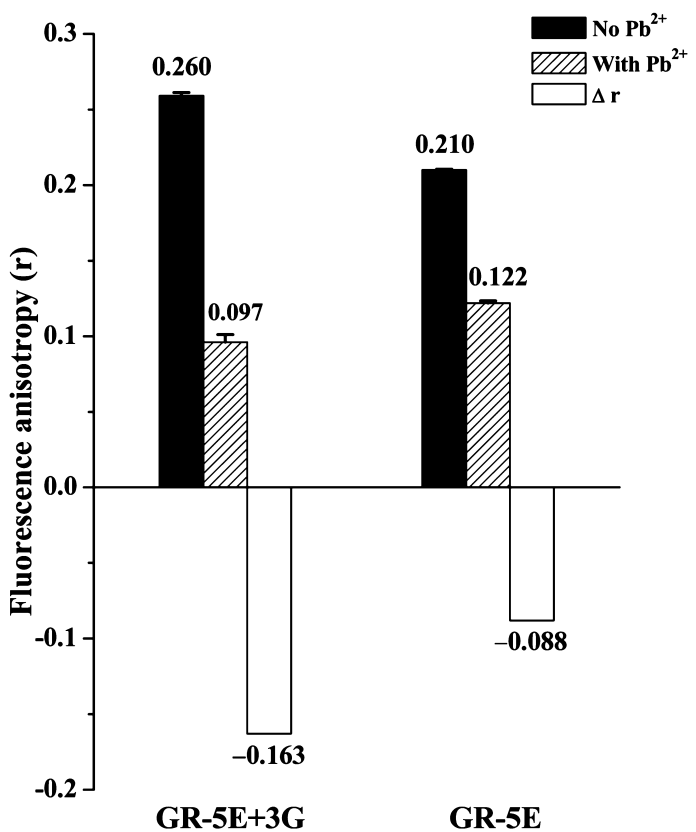

Figure 1. Fluorescence anisotropy of GR-5 DNAzymes $(20 \mathrm{nM})$ in the absence (black bars) and presence of (hatched bars) of $\mathrm{Pb}^{2+}(200$ $\mathrm{nM})$. The DNAzyme solutions were prepared with the buffer of 50 $\mathrm{mM}$ Tris-HAc pH 7.5, $200 \mathrm{mM} \mathrm{NaCl}$, and fluorescence anisotropy was measured after $2 \mathrm{~h}$ incubation at room temperature. The measurements were conducted in triplicate.

$5 \mathrm{E}+3 \mathrm{G})$, the FA response $(r)$ was further increased to 0.260 as expected. As stimulated by $200 \mathrm{nM} \mathrm{Pb}^{2+}$, the FA response $(r)$ decreased to 0.122 for GR-5E/GR-5S-TMR and 0.097 for GR5E+3G/GR-5S-TMR, respectively. In essence, the fluorescence anisotropy of the $2 \mathrm{~h}$ cleaving GR-5E+3G/GR-5S-TMR system $(r=0.097)$ is slightly higher than that of the pure TMR-labeled 5-nucleotides fragment (GR-5P-TMR, $r=0.080$; Table 1). This suggests that most of the substrate GR-5S-TMR was cleaved in the second DNAzyme system within $2 \mathrm{~h}$. Unexpectedly, without the assistance of tandem G bases in the first DNAzyme system, the fluorescence anisotropy after $2 \mathrm{~h}$ cleaving displayed a much higher value $(r=0.122)$. Probably, the cleavage in the first DNAzyme system is incomplete. These results support that the tandem $G$ bases can be used as a FA enhancer by interacting with the fluorophore TMR and probably promoting DNAzyme cleavage activity.

Optimization of the DNAzyme Activity Assay. To optimize the FA assay for detection of DNAzyme activity, we investigated the effects of the number of the tandem $\mathrm{G}$ base of the DNAzyme strand, incubation time, buffer $\mathrm{pH}$, and ion strength. As shown in Figure 2A, the absolute FA change obviously increased with the increasing number of tandem $G$ base from 1 to 3 , compared to that without any extended $G$ base at the $3^{\prime}$-end of the DNAzyme strand. However, it decreased with further introducing additional $G$ base(s). The maximum reduction in FA was $\Delta r=-0.160$ for including three tandem $\mathrm{G}$ bases at the $3^{\prime}$-end of the GR-5E strand (GR-5E $+3 G)$. Therefore, in the following cases, we only tested the FA response of GR-5E+3G/GR-5S-TMR in the presence of 200 $\mathrm{nM} \mathrm{Pb}{ }^{2+}$. As shown in Figure $2 \mathrm{~B}$, the FA of the hybridized GR5E+3G/GR-5S-TMR gradually decreased from $r=0.260$ to $r=$ $0.098(\Delta r=-0.162)$ with increasing incubation time from zero to $120 \mathrm{~min}$. No further decrease in FA was observed for longer 

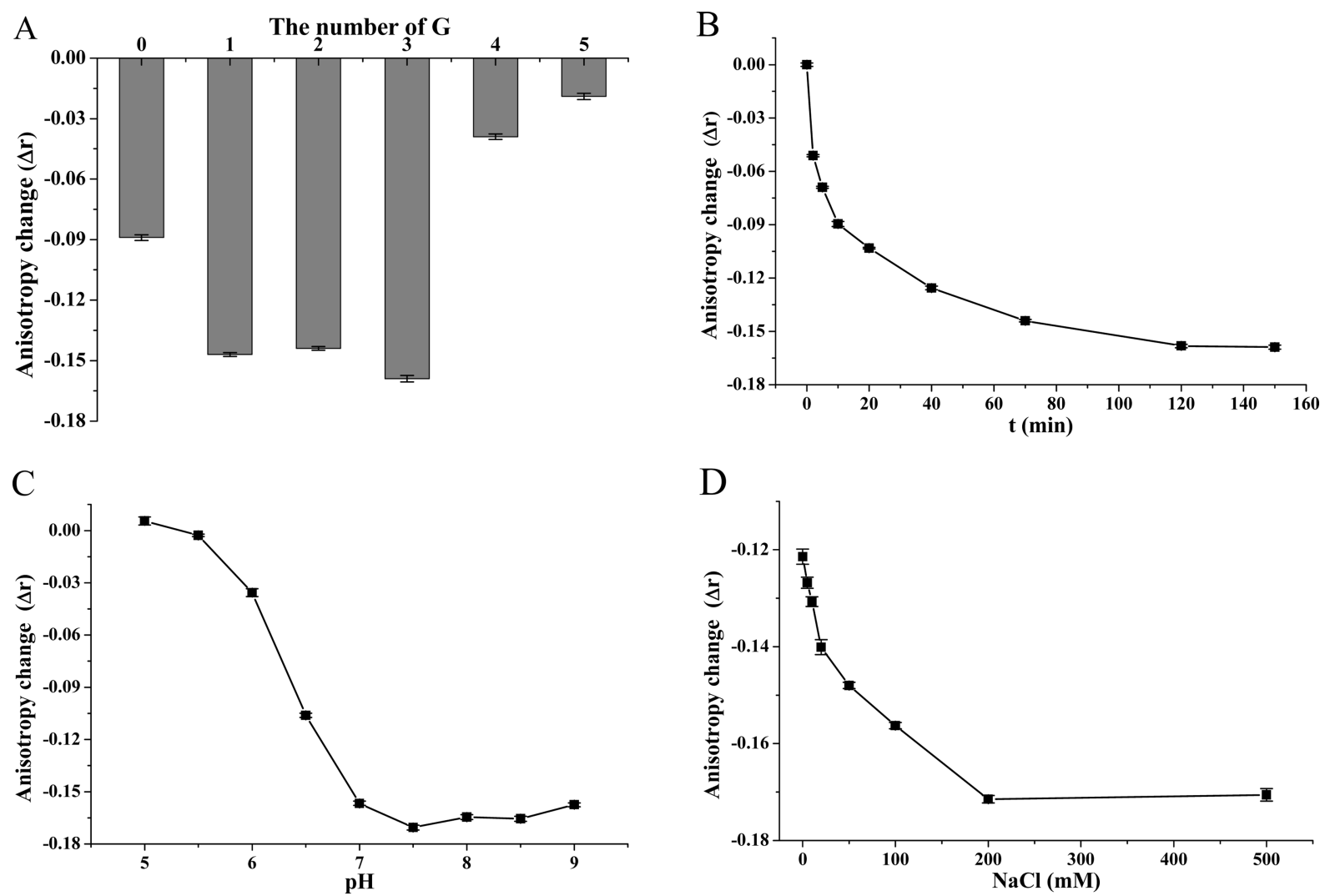

Figure 2. (A) Effect of the number of extended G base at the $3^{\prime}$-end of the GR-5E ( $\left.20 \mathrm{nM}\right)$ on the FA change of GR-5S-TMR (20 nM) in the presence of $200 \mathrm{nM} \mathrm{Pb}^{2+}$. Effects of incubation time (B), $\mathrm{pH}(\mathrm{C})$, and ion strength (D) on the fluorescence anisotropy change $(\Delta r)$ of GR-5E+3G/ GR-5S-TMR $(20 \mathrm{nM})$ in the presence of $200 \mathrm{nM} \mathrm{Pb}^{2+}$. The experimental condition is the same as that in Figure 1.

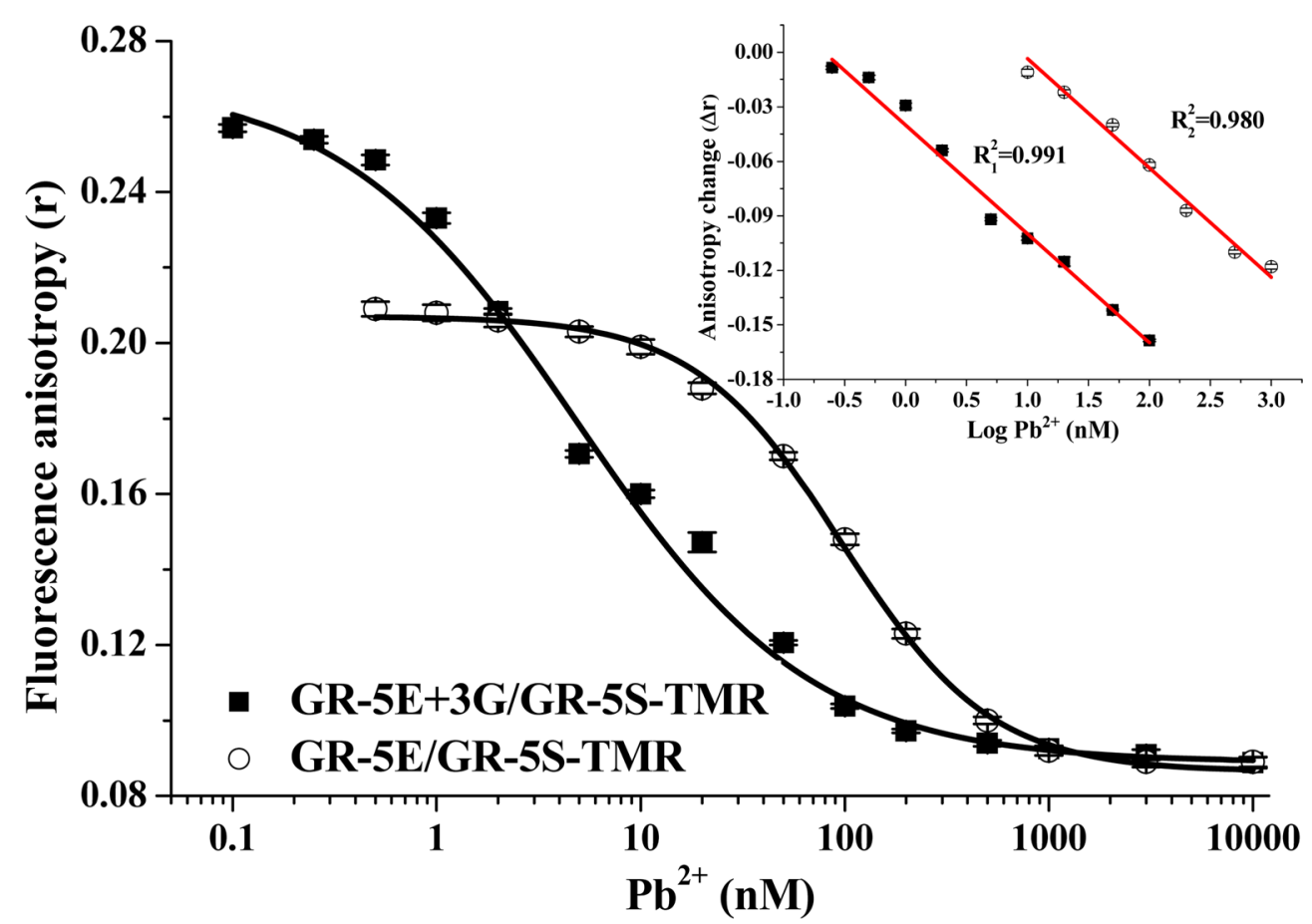

Figure 3. Fluorescence anisotropy of GR-5E+3G/GR-5S-TMR and GR-5E/GR-5S-TMR bimolecular assembly titrated with increasing concentrations of $\mathrm{Pb}^{2+}$. The inset shows the linear relationship between the change in fluorescence anisotropy $(\Delta r)$ and the logarithm of the $\mathrm{Pb}^{2+}$ concentration range of $0.2-100 \mathrm{nM}$ for GR-5E+3G/GR-5S-TMR and 10-100 nM for GR-5E/GR-5S-TMR bimolecular assembly. The experimental condition is the same as that in Figure 1. 
incubation. Therefore, FA measurement was performed after 120 min incubation.

The FA change is also sensitive to the pH. FA did not decrease when the $\mathrm{pH}$ of the binding buffer solution was lower than 5.5 (Figure 2C), indicating a loss of the cleavage activity of the GR-5E+3G/GR-5S-TMR at $\mathrm{pH}<5.5$. However, the absolute FA change sharply increased to 0.170 with an increasing $\mathrm{pH}$ from 5.5 to 7.5 and became leveled off with further increasing $\mathrm{pH}$. Accordingly, a buffer solution of $\mathrm{pH} 7.5$ was selected as the buffer for GR-5 DNAzyme activity assays.

Furthermore, the absolute FA change $(|\Delta r|$, see Figure 2D) gradually increased with the increasing concentration of $\mathrm{Na}^{+}$up to $200 \mathrm{mM}$ and then leveled off. The maximum reduction in FA of GR-5E+3G/GR-5S-TMR was $\Delta r=-0.168$ in the presence of $200-500 \mathrm{mM} \mathrm{NaCl}$. The presence of a high concentration of $\mathrm{Na}^{+}$is probably helpful to the formation of the stably hybridized assembly and/or to enhance the DNAzyme activity to cleave the substrate.

Specific Detection of Cofactor $\mathrm{Pb}^{2+}$ by GR5 DNAzyme Activity Assay. Although it is developed for detection of GR-5 DNAzyme activity, our approach is also possible for detection of free $\mathrm{Pb}^{2+}$. This is because the catalytic activity of the GR-5 DNAzyme cleaving rA site is dependent on the cofactor $\mathrm{Pb}^{2+}$. As shown in Figure 3, the FA signal decreased in a $\mathrm{Pb}^{2+}$ concentration-dependent manner. In contrast, no significant FA change was observed for the other nine divalent metal ions with 1000 -fold excess amount $\left(\mathrm{Ca}^{2+}, \mathrm{Mg}^{2+}, \mathrm{Co}^{2+}, \mathrm{Ni}^{2+}, \mathrm{Mn}^{2+}, \mathrm{Cu}^{2+}\right.$, $\mathrm{Sr}^{2+}, \mathrm{Ba}^{2+}$, and $\mathrm{Cd}^{2+}$; Figure $\left.4 \mathrm{~A}\right)$. Suggesting the tested divalent metal ions other than $\mathrm{Pb}^{2+}$ cannot function as a cofactor to endow a rA cleaving activity to the GR-5 DNAzyme. Furthermore, the coexistence of $\mathrm{Ca}^{2+}, \mathrm{Mg}^{2+}, \mathrm{Co}^{2+}, \mathrm{Ni}^{2+}$, $\mathrm{Mn}^{2+}, \mathrm{Cu}^{2+}, \mathrm{Sr}^{2+}, \mathrm{Ba}^{2+}$, and $\mathrm{Cd}^{2+}$ cannot impair the cleavage activity of GR-5 DNAzyme (Figure 4B). These results suggest that the tested divalent metal ions cannot interfere with the binding of $\mathrm{Pb}^{2+}$ to the GR-5 DNAzyme, which is critical for the cleavage activity of the DNAzyme. ${ }^{38,44,45}$ All of these results support that the GR-5 DNAzyme activity assay can be exploited to specifically detect free $\mathrm{Pb}^{2+}$ in the aqueous solution.

The dynamic range for the detection of free $\mathrm{Pb}^{2+}$ is about 3 orders of magnitude for GR-5E+3G/GR-5S-TMR (0.2-100 $\mathrm{nM}$; see inset of Figure 3) and 2 orders of magnitude for GR5E/GR-5S-TMR (10-1000 nM; see inset of Figure 3). On the basis of a signal-to-noise $(S / N)$ ratio of 3 , the limit of detection (LOD) for $\mathrm{Pb}^{2+}$ was $0.1 \mathrm{nM}$ to GR-5E+3G/GR-5S-TMR, while the LOD was $2 \mathrm{nM}$ to GR-5E/GR-5S-TMR. The results demonstrate that the DNAzyme with extending $G$ bases displays 20 times higher sensitivity than no-extended Gcontaining DNAzyme.

Characterization of the Interactions of $\mathrm{Pb}^{2+}$ and Free Thiol-Containing Molecules. In addition to the application to the detection of free $\mathrm{Pb}^{2+}$ in aqueous solution, it is also possible for the measurement of the GR-5 DNAzyme activity to characterize the interactions of biomolecules with $\mathrm{Pb}^{2+}$. We took free thiol-containing molecules cysteine (Cys) and glutathione $(\mathrm{GSH})$ as examples. Both Cys and GSH play a crucial role in the human body ${ }^{46}$ and show strong interaction with $\mathrm{Pb}^{2+} \cdot{ }^{47}$ We expected that the binding of $\mathrm{Pb}^{2+}$ to free thiolcontaining molecules would deprive $\mathrm{Pb}^{2+}$ from the GR-5 DNAzyme and reduce its rA-cleaving activity (Figure 5A). Indeed, both $\mathrm{Cys}$ and GSH can diminish the $\mathrm{Pb}^{2+}$-dependent rA-cleaving activity as manifested by the no change in FA (Figure 5B).
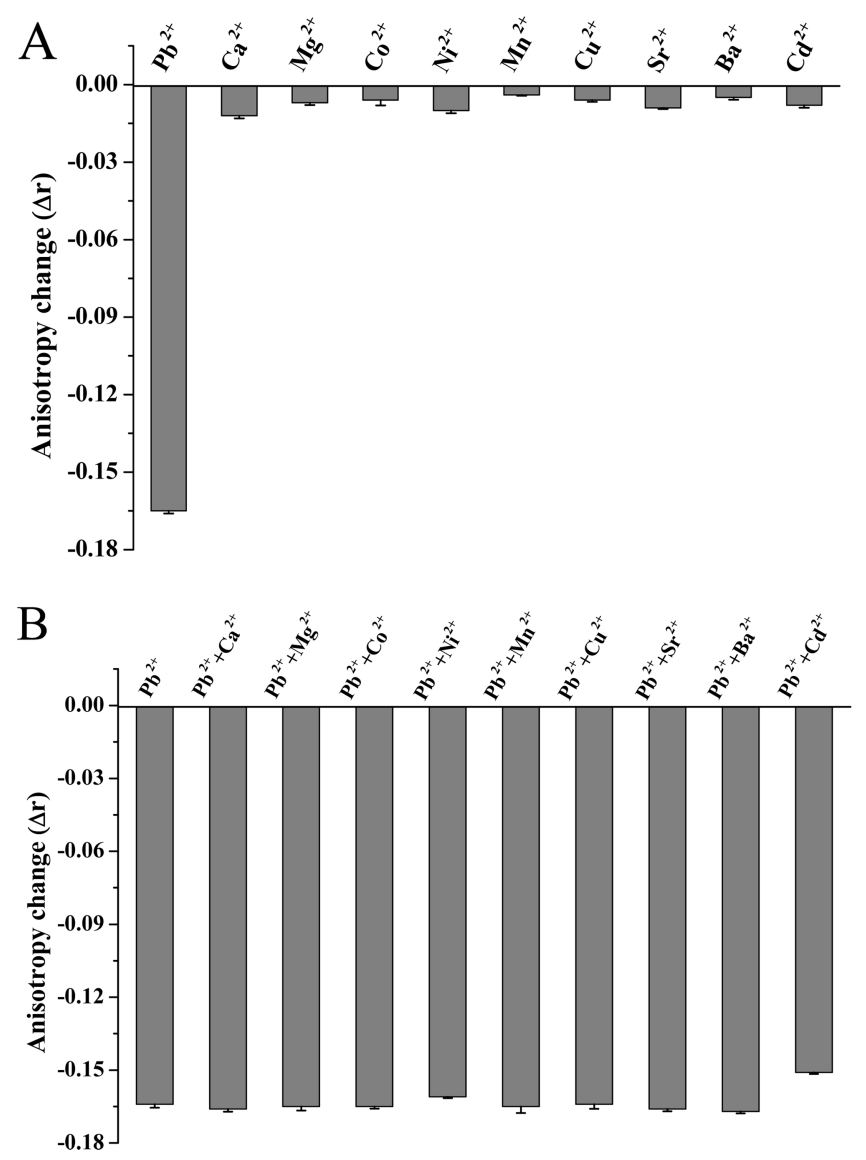

Figure 4. Fluorescence anisotropy change $(\Delta r)$ of GR-5E+3G/GR-5STMR bimolecular assembly (A) in the presence of $200 \mathrm{nM} \mathrm{Pb}^{2+}$ or $200 \mu \mathrm{M}$ other metal ions $\left(\mathrm{Ca}^{2+}, \mathrm{Mg}^{2+}, \mathrm{Co}^{2+}, \mathrm{Ni}^{2+}, \mathrm{Mn}^{2+}, \mathrm{Cu}^{2+}, \mathrm{Sr}^{2+}\right.$, $\mathrm{Ba}^{2+}$, and $\mathrm{Cd}^{2+}$ ) and (B) in the presence of both $200 \mathrm{nM} \mathrm{Pb}^{2+}$ and 200 $\mu \mathrm{M}$ other metal ions. The experimental condition is the same as that in Figure 1.

In detail, the FA value significantly increased from $r=0.097$ to $r=0.262$ when $5.0 \mu \mathrm{M}$ Cys was added to the hybridized GR5E+3G/GR-5S-TMR system (Figure 5B), which is close to the FA value of the hybridized bimolecular assembly $(r=0.263)$. The result suggests that the cleaving activity of the GR-5 DNAzyme is markedly inhibited by Cys. Essentially, the inhibition is also Cys-concentration-dependent (Figure 6). A good linear relationship was observed to the concentration of Cys ranging from 50 to $500 \mathrm{nM}$. The LOD for Cys was $20 \mathrm{nM}$ based on a $S / N=3$. In contrast, seven other amino acids that do not contain any free thiol cannot inhibit the $\mathrm{Pb}^{2+}$-dependent GR-5 DNAzyme activity (Figure 5B).

Similarly, GSH, a widely existing tripeptide in living organisms, ${ }^{48}$ can also inhibit the $\mathrm{Pb}^{2+}$-dependent GR-5 DNAzyme activity in a concentration-dependent manner (Figures 5 and 6). However, the inhibition efficiency of GSH $\left(\mathrm{IC}_{50}=65 \mu \mathrm{M}\right)$ is about 200 times weaker than that of Cys $\left(\mathrm{IC}_{50}=0.32 \mu \mathrm{M}\right)$. The results suggest that the free thiol of Cys is easier to complex with $\mathrm{Pb}^{2+}$ than that of GSH. This is consistent with previous work showing the higher complexing capability of Cys than GSH. ${ }^{49}$

In this work, we developed a fluorescence anisotropy assay for monitoring GR-5 DNAzyme activity. Interestingly, we found that the extension of the $3^{\prime}$-termini of the DNAzyme strand with three tandem $G$ bases significantly enhanced FA change upon the rA site cleaving. Unexpectedly, we also found 

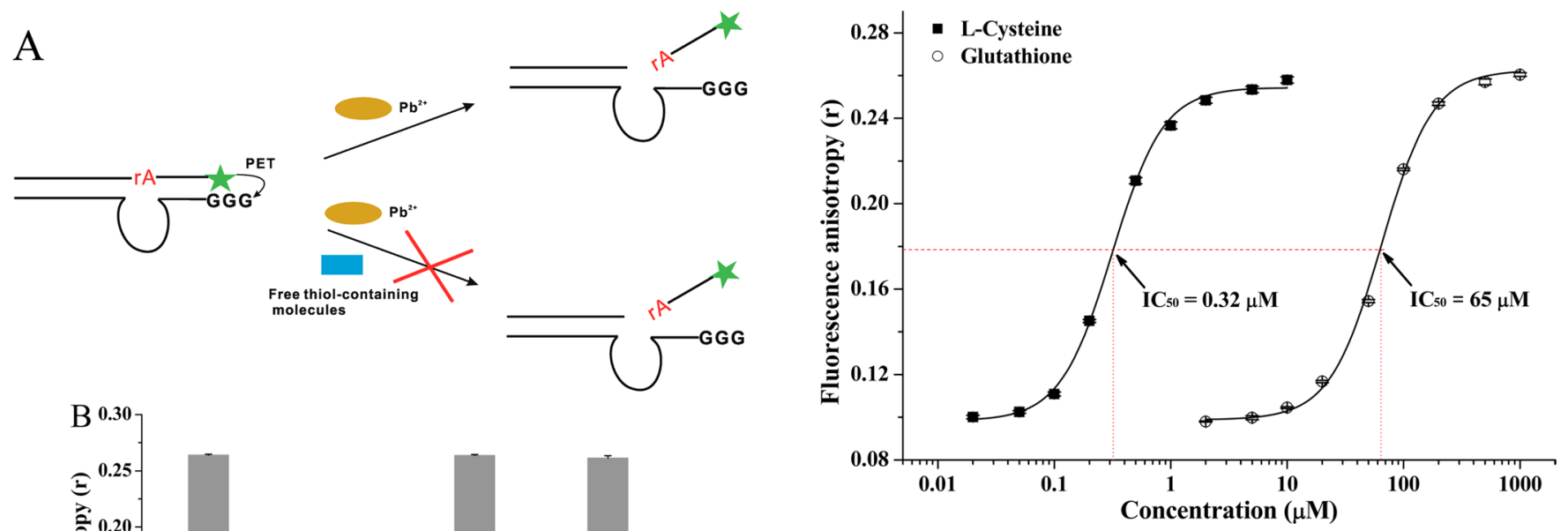

Figure 6. Evaluation of the interaction of $\mathrm{Pb}^{2+}$ and free thiolcontaining molecules by measuring GR5 DNAzyme activity. The solutions contained GR-5E+3G/GR-5S-TMR, $100 \mathrm{nM} \mathrm{Pb}^{2+}$, and cysteine or glutathione of varying concentration.

free $\mathrm{Pb}^{2+}$ is critical to the lead toxicity. Therefore, the detection of free $\mathrm{Pb}^{2+}$ is of intensive interest. The common methods, atomic absorption spectroscopy (AAS), ${ }^{52}$ inductively coupled plasma optical emission spectroscopy (ICP-OES), ${ }^{53}$ and inductively coupled plasma mass spectrometry (ICP-MS), ${ }^{54}$ can detect $\mathrm{Pb}^{2+}$ at the levels of $0.50 \mathrm{ng} / \mathrm{L}$ to $50 \mu \mathrm{g} / \mathrm{L}(2 \mathrm{pM}$ to $242 \mathrm{nM})$. However, these methods suffer from high cost, sophisticated instruments, and complicated sample pretreatment, making it difficult for real-time and on-site monitoring of lead ion. A number of fluorescent $\mathrm{Pb}^{2+}$ sensors have been developed, which are based on the fluorescently labeled small organic chelators, ${ }^{55,56}$ organic polymers, ${ }^{57}$ peptides, ${ }^{58}$ and functional nucleic acid. ${ }^{3,9}$ The detection limit of our nonnanoparticle-dependent FA approach (100 pM) is lower than that of nanoparticle-dependent FA approaches (LOD, $1 \mathrm{nM}$, $)^{18}$ and other fluorescence intensity-based homogeneous analysis (LOD, $3.7 \mathrm{nM})^{38}$

Our approach for detection of free $\mathrm{Pb}^{2+}$ has several

Figure 5. (A) Schematic representation of thiol-containing molecule inhibiting the catalytic activity of DNAzyme. The red $\times$ represents inhibiting the catalytic activity of DNAzyme. (B) Fluorescence anisotropy of different sensing systems: DNAzyme, DNAzyme + $\mathrm{Pb}^{2+}$; DNAzyme + Cys $+\mathrm{Pb}^{2+}$; DNAzyme $+\mathrm{GSH}+\mathrm{Pb}^{2+}$. (C) Fluorescence anisotropy change $(\Delta r)$ of GR-5E+3G/GR-5S-TMR bimolecular assembly in the presence of $200 \mathrm{nM} \mathrm{Pb}^{2+}$ and L-cysteine $(2 \mu \mathrm{M})$ or other amino acids $(200 \mu \mathrm{M})$. The experimental condition is the same as that in Figure 1.

that the tandem $\mathrm{G}$ bases could increase the $\mathrm{rA}$ site cleaving activity, by comparing the FA value of the cleaved products with that of the pure TMR-labeled fragment with the identical sequence from the cleaved product.

Benefiting from the improved FA assay, a large FA change $(|\Delta r|=0.170)$ can be obtained upon the GR-5 DNAzyme catalytic activity. The nine divalent metal ions $\left(\mathrm{Ca}^{2+}, \mathrm{Mg}^{2+}\right.$, $\mathrm{Co}^{2+}, \mathrm{Ni}^{2+}, \mathrm{Mn}^{2+}, \mathrm{Cu}^{2+}, \mathrm{Sr}^{2+}, \mathrm{Ba}^{2+}$, and $\left.\mathrm{Cd}^{2+}\right)$ being present at 1000-times higher concentration cannot interfere with the interaction of $\mathrm{Pb}^{2+}$ and GR-5 DNAzyme. By the application of our developed GR-5 DNAzyme activity assay, we could detect $100 \mathrm{pM}$ free $\mathrm{Pb}^{2+}$ in aqueous solution.

In fact, lead is one of the abundant and toxic heavy metals ${ }^{50}$ and can cause neurological, reproductive, cardiovascular, and developmental disorders, slowed motor responses and decreased IQs particularly in children. ${ }^{51}$ The concentration of advantages. First, it only requires fluorescently labeling of one end of the DNA strand and introducing guanine bases near the fluorophore TMR. Second, the assay is quite simple, rapid, and convenient. It only needs to mix the substrate probe, the enzyme strand, and the target molecule in homogeneous solution. No troublesome separation or immobilization procedures are required. Third, this approach may also be extended to other target molecules detection by simply switching the corresponding DNAzyme. Finally, the assay can be easily carried out in 96- or 384-well plates, rendering it amenable to high-throughput application. On the other hand, this technology is potentially useful for analyzing other DNAzyme activity and detection of other target/cofactor or inhibitors of similar functional nucleic acids.

Interestingly, by monitoring the GR-5 DNAzyme activity, the interaction of $\mathrm{Pb}^{2+}$ - and free-thiol-containing molecules can be evaluated. By this approach, we showed that $\mathrm{Pb}^{2+}$ preferentially complexes with single amino acid cysteine than with tripeptide glutathione.

\section{CONCLUSION}

In summary, we developed a non-nanoparticle-dependent FA amplification approach for detection of $\mathrm{Pb}^{2+}$-dependent GR-5 DNAzyme activity. The attachment of additional G bases in the 
DNAzyme strand could introduce intramolecular interaction between fluorophore TMR and the spatially close guanine bases and probably promote the DNAzyme cleavage activity. By this design, a remarkable FA change upon cleavage of TMRlabeled substrate by DNAzyme was achieved. This improved FA analysis was used to real-time monitor the cleavage activity of DNAzyme and to quantify its cofactor $\mathrm{Pb}^{2+}$, and to evaluate the interaction of $\mathrm{Pb}^{2+}$ with free thiols.

\section{AUTHOR INFORMATION}

\section{Corresponding Author}

*Fax: +86-10-62849600. Tel.: +86-10-62849600. E-mail: hlwang@rcees.ac.cn.

\section{Notes}

The authors declare no competing financial interest.

\section{ACKNOWLEDGMENTS}

This work was supported by grants from the National Basic Research Program of China (2015CB932003), the National Natural Science Foundation of China (21305153 to D.Z.; 21327006, 21125523, and 21435008 to H.W.), and the Strategic Priority Research Program of the Chinese Academy of Sciences (XBD14030200 to H.W.).

\section{REFERENCES}

(1) Dass, C. R.; Choong, P. F. M.; Khachigian, L. M. Mol. Cancer Ther. 2008, 7, 243-252.

(2) Benson, V. L.; Khachigian, L. M.; Lowe, H. C. Br. J. Pharmacol. 2008, 154, 741-748.

(3) Liu, J.; Cao, Z.; Lu, Y. Chem. Rev. 2009, 109, 1948-1998.

(4) Willner, I.; Shlyahovsky, B.; Zayats, M.; Willner, B. Chem. Soc. Rev. 2008, 37, 1153-1165.

(5) Sen, D.; Geyer, C. R. Curr. Opin. Chem. Biol. 1998, 2, 680-687.

(6) Lan, T.; Lu, Y. In Interplay between metal ions and nucleic acids, Metal ions in life sciences, Vol. 10; Sigel, A., Sigel, H., Sigel, R. K. O., Eds.; Springer Science+Business Media B. V.: New York, 2012; pp 217-248.

(7) Lu, Y. Chem.-Eur. J. 2002, 8, 4588-4596.

(8) Sando, S.; Sasaki, T.; Kanatani, K.; Aoyama, Y. J. Am. Chem. Soc. 2003, 125, 15720-15721.

(9) Zhang, X. B.; Kong, R. M.; Lu, Y. Annu. Rev. Anal. Chem. 2011, 4, $105-128$.

(10) Lu, Y.; Liu, J.; Li, J.; Bruesehoff, P. J.; Pavot, C. M. B.; Brown, A. K. Biosens. Bioelectron. 2003, 18, 529-540.

(11) Navani, N. K.; Li, Y. Curr. Opin. Chem. Biol. 2006, 10, 272-281.

(12) Elbaz, J.; Lioubashevski, O.; Wang, F.; Remacle, F.; Levine, R.

D.; Willner, I. Nat. Nanotechnol. 2010, 5, 417-422.

(13) Stojanovic, M. N.; Mitchell, T. E.; Stefanovic, D. J. Am. Chem. Soc. 2002, 124, 3555-3561.

(14) Santoro, S. W.; Joyce, G. F. Proc. Natl. Acad. Sci. U. S. A. 1997, 94, 4262-4266.

(15) Brown, A. K.; Li, J.; Pavot, C. M. B.; Lu, Y. Biochemistry 2003, $42,7152-7161$.

(16) Liu, J.; Lu, Y. J. Am. Chem. Soc. 2002, 124, 15208-15216.

(17) Kim, H. K.; Rasnik, I.; Liu, J.; Ha, T.; Lu, Y. Nat. Chem. Biol. 2007, 3, 763-768.

(18) Yin, B. C.; Zuo, P.; Huo, H.; Zhong, X.; Ye, B.-C. Anal. Biochem. 2010, 401, 47-52.

(19) Yu, Y.; Liu, Y.; Zhen, S. J.; Huang, C. Z. Chem. Commun. (Cambridge, U. K.) 2013, 49, 1942-1944.

(20) Amaral, N. B.; Zuliani, S.; Guieu, V.; Ravelet, C.; Perrier, S.; Peyrin, E. Anal. Bioanal. Chem. 2014, 406, 1173-1179.

(21) He, Y.; Tian, J.; Zhang, J.; Chen, S.; Jiang, Y.; Hu, K.; Zhao, Y.; Zhao, S. Biosens. Bioelectron. 2014, 55, 285-288.

(22) Wang, H. B.; Wang, L.; Huang, K. J.; Xu, S. P.; Wang, H. Q.; Wang, L. L.; Liu, Y. M. New. J. Chem. 2013, 37, 2557-2563.
(23) Jameson, D. M.; Ross, J. A. Chem. Rev. 2010, 110, 2685-2708. (24) Checovich, W. J.; Bolger, R. E.; Burke, T. Nature 1995, 375, 254-256.

(25) Kwok, P. Y. Hum. Mutat. 2002, 19, 315-323.

(26) LiCata, V. J.; Wowor, A. J. Methods Cell Biol. 2008, 84, 243-262.

(27) Smith, D. S.; Eremin, S. A. Anal. Bioanal. Chem. 2008, 391, 1499-1507.

(28) Goulko, A. A.; Zhao, Q.; Guthrie, J. W.; Zou, H.; Le, X. C. Springer Ser. Fluoresc. 2008, 5, 303-322.

(29) Wang, H.; Lu, M.; Tang, M. S.; Houten, B. V.; Ross, J. B. A.; Weinfeld, M.; Le, X. C. Proc. Natl. Acad. Sci. U. S. A. 2009, 106, 12849-12854.

(30) Lakowicz, J. R. Principles of fluorescence spectroscopy, 3rd ed.; Springer-Verlag: Berlin, Heidelberg, 2006.

(31) Li, T.; Zhang, D.; Luo, W.; Lu, M.; Song, Y.; Wang, H. Anal. Chem. 2010, 82, 487-490.

(32) Zhang, D.; Lu, M.; Wang, H. J. Am. Chem. Soc. 2011, 133, 9188-9191.

(33) Zhang, D.; Zhao, Q.; Zhao, B.; Wang, H. Anal. Chem. 2012, 84, 3070-3074.

(34) Zhang, D.; Shen, H.; Li, G.; Zhao, B.; Yu, A.; Zhao, Q.; Wang, H. Anal. Chem. 2012, 84, 8088-8094.

(35) Zhang, D.; Yin, L.; Meng, Z.; Yu, A.; Guo, L.; Wang, H. Anal. Chim. Acta 2014, 812, 161-167.

(36) Zhao, Q.; Lv, Q.; Wang, H. Anal. Chem. 2014, 86, 1238-1245.

(37) Breaker, R. R.; Joyce, G. F. Chem. Biol. 1994, 1, 223-229.

(38) Lan, T.; Furuya, K.; Lu, Y. Chem. Commun. (Cambridge, U. K.) 2010, 46, 3896-3898.

(39) Qu, P.; Chen, X.; Zhou, X.; Li, X.; Zhao, X. Sci. China, Ser. B: Chem. 2009, 52, 1653-1659.

(40) Heinlein, T.; Knemeyer, J. P.; Piestert, O.; Sauer, M. J. Phys. Chem. B 2003, 107, 7959-7964.

(41) Maruyama, N.; Shinobara, T.; Ichinose, H.; Kitaoka, M.; Okamura, N.; Kamiya, N.; Goto, M. Biotechnol. Lett. 2005, 27, 13491354.

(42) Edman, L.; Metes, U.; Rig, R. Proc. Natal. Acad. Acad. Sci. U. S. A. 1996, 93, 6710-6715.

(43) Torimura, M.; Kurata, S.; Yamada, K.; Yokomaku, T.; Kamagata, Y.; Kanagawa, T.; Kurane, R. Anal. Sci. 2001, 17, 155-160.

(44) Nie, D.; Wu, H.; Zheng, Q.; Guo, L.; Ye, P.; Hao, Y.; Li, Y.; Fu, F.; Guo, Y. Chem. Commun. (Cambridge, U. K.) 2012, 48, 1150-1152. (45) Zhao, X. H.; Kong, R. M.; Zhang, X. B.; Meng, H. M.; Liu, W. N.; Tan, W.; Shen, G. L.; Yu, R. Q. Anal. Chem. 2011, 83, 5062-5066. (46) Droge, W.; Holm, E. FASEB J. 1997, 11, 1077-1089.

(47) Magyar, J. S.; Weng, T. C.; Stern, C. M.; Dye, D. F.; Rous, B. W.; Payne, J. C.; Bridgewater, B. M.; Mijovilovich, A.; Parkin, G.; Zaleski, J. M.; Penner-Hahn, J. E.; Godwin, H. A. J. Am. Chem. Soc. 2005, 127, 9495-9505.

(48) Sies, H. Free Radical Biol. Med. 1999, 27, 916-921.

(49) Almodhefer, A. J. A.; Bradbury, M. W. B.; Simons, T. J. B. Clin. Sci. 1991, 81, 823-829.

(50) Flegal, A. R.; Smith, D. R. Environ. Res. 1992, 58, 125-133.

(51) Needleman, H. Annu. Rev. Med. 2004, 55, 209-222.

(52) Liu, Y.; Liu, Z.; Wang, Y.; Dai, J.; Gao, J.; Xie, J.; Yan, Y. Microchim. Acta 2011, 172, 309-317.

(53) Korn, M. G. A.; Andrade, J. B. de; Jesus, D. S. de; Lemos, V. A.; Bandeira, M. L. S. F.; dos Santos, W. N. L.; Bezerra, M. A.; Amorim, F. A. C.; Souza, A. S.; Ferreira, S. L. C. Talanta 2006, 69, 16-24.

(54) Schütz, A.; Bergdahl, I. A.; Ekholm, A.; Skerfving, S. Occup. Environ. Med. 1996, 53, 736-740.

(55) Kwon, J. Y.; Jang, Y. J.; Lee, Y. J.; Kim, K. M.; Seo, M. S.; Nam, W.; Yoon, J. J. Am. Chem. Soc. 2005, 127, 10107-10111.

(56) Kim, S. K.; Lee, S. H.; Lee, J. Y.; Lee, J. Y.; Bartsch, R. A.; Kim, J. S. J. Am. Chem. Soc. 2004, 126, 16499-16506.

(57) Kim, I. K.; Dunkhorst, A.; Gilbert, J.; Bunz, U. H. F. Macromolecules 2005, 38, 4560-4562.

(58) Deo, S.; Godwin, H. A. J. Am. Chem. Soc. 2000, 122, 174-175. 